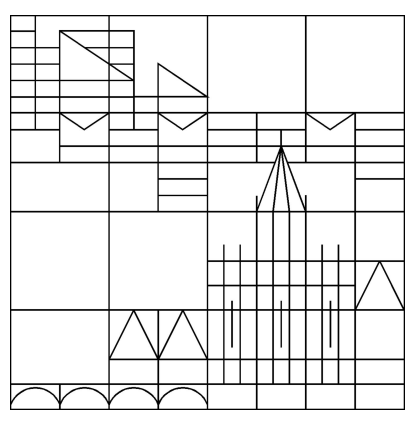

\title{
A space mapping approach for the $p$-Laplace equation
}

\author{
Oliver Lass \\ Stefan Volkwein
}

Konstanzer Schriften in Mathematik

(vormals: Konstanzer Schriften in Mathematik und Informatik)

Nr. 258, November 2009

ISSN $1430-3558$ 



\title{
A Space Mapping Approach for the $p$-Laplace Equation
}

\author{
Oliver Lass ${ }^{1}$ and Stefan Volkwein ${ }^{2}$
}

1 Fachbereich Mathematik und Statistik, Universität Konstanz, Universitätsstraße 10, D-78457 Konstanz, Germany. oliver.lass@uni-konstanz.de

2 Fachbereich Mathematik und Statistik, Universität Konstanz, Universitätsstraße 10, D-78457 Konstanz, Germany. stefan.volkwein@uni-konstanz.de

\section{Introduction}

A main aspect in the design of passenger cars with respect to pedestrian safety is the energy absorption capability of the car parts. Besides that, the car parts have to fulfill several other requirements. The associated optimal problem is hard to solve numerically. That makes it necessary to develop easy and fast to solve prediction models with little loss in accuracy for optimization purpose. Current simulation tools combined with standard optimization software are not well suited to deal with the above mentioned needs [13].

We will show the application of mathematical methods on a simplified model to reduce the optimization effort. The goal of the structural optimization problem (see $[7,8]$ ) is to determine a thickness parameter $\lambda$ of a plate $\Omega \subset \mathbb{R}^{2}$ (representing a part of the vehicle) and an associated displacement $u$ satisfying the nonlinear $p$-Laplace equation

$$
-\operatorname{div}\left(2(1+n) \lambda(\mathbf{x})|\nabla u(\mathbf{x})|_{2}^{2 n} \nabla u(\mathbf{x})\right)=g(\mathbf{x}) \quad \text { for all } \mathbf{x} \in \Omega
$$

together with Dirichlet boundary conditions, where $g$ represents a force acting on $\Omega, n \in(0,1)$ is the Hollomon coefficient, and $|\cdot|_{2}$ stands for the Euclidean norm. We suppose that $0<\lambda_{a} \leq \lambda(\mathbf{x}) \leq \lambda_{b}$ with positive scalars $\lambda_{a}$ and $\lambda_{b}$. Our goal is to minimize the mass of the plate, i.e., to minimize the integral

$$
J_{1}(\lambda)=\int_{\Omega} \lambda(\mathbf{x}) \mathrm{d} \mathbf{x}
$$

but also to avoid that the displacement is larger than a given threshold $u_{b}>0$. This issue is motivated by our pedestrian safety application. Thus we choose

$$
J_{2}(u)=\beta \int_{\Omega} \min \left(u(\mathbf{x})-u_{b}(\mathbf{x}), 0\right)^{3} \mathrm{~d} \mathbf{x}
$$

as the second part of our cost functional. Here $\beta>0$ is a weighting parameter. Due to the nonlinear structure of the elliptic partial differential equation, the 
numerical solution of the optimization problem governed by the partial differential equation (PDE) constraint (1) is expensive, we consider an alternative constraint given by

$$
-\operatorname{div}(2(1+n) \mu(\mathbf{x}) \nabla v(\mathbf{x}))=g(\mathbf{x}) \quad \text { for all } \mathbf{x} \in \Omega,
$$

which is a linear elliptic PDE. We will call (1) the fine model and (2) the coarse model. It turns out that the space-mapping technique [9] provides an attractive framework to improve the use of the coarse model as a surrogate for the optimization of the fine model. The space mapping technique is utilized to link the linear and nonlinear equations and drives the optimization iteration of the time intensive nonlinear equation using the fast linear equation. For this reason an efficient realization of the space mapping is utilized.

The space mapping technique was first introduced in [2]. The idea of the space mapping has been developed along different directions and generalized to a number of contexts [14]. One of the problems lies in the information necessary to compute the Jacobian of the space mapping which involves expensive gradient information of (1). In [1] Broyden's method is utilized to construct an approximation of the Jacobian. This approach will be presented. In the context of PDEs, we refer to $[6,10]$. Compared to $[1,2,14]$, our modified approach is similar to [6], where a modified Broyden formula is used.

The paper is organized in the following manner. In Section 2 we introduce the infinite-dimensional optimization problem for the $p$-Laplace equation. The space mapping approach is described in Section 3, whereas in Section 4 the surrogate optimization problem is formulated. Section 5 is devoted to present numerical examples illustrating the advantage of the proposed approach.

\section{Optimization of the complex model}

In this section we formulate optimal control problem governed by the $p$ Laplace equation. By $W_{0}^{1, p}(\Omega), p \in[1, \infty)$, we denote the Sobolev space of weakly differentiable functions, whose weak derivative belongs to $L^{q}(\Omega)$ and whose function values are zero on the boundary $\Gamma=\partial \Omega$. We set $p=2 n+2$ for $n \in(0,1)$. Let us define the Banach space $X=L^{\infty}(\Omega) \times\left(W_{0}^{1, p}(\Omega) \cap L^{\infty}(\Omega)\right)$ and the nonlinear operator $f: X \rightarrow W_{0}^{1, p}(\Omega)^{\prime}$ (fine model) as

$\langle f(x), \varphi\rangle_{\left(W_{0}^{1, p}\right)^{\prime}, W_{0}^{1, p}}=\int_{\Omega} 2(1+n) \lambda(\mathbf{x})|\nabla u(\mathbf{x})|_{2}^{p-2} \nabla u(\mathbf{x}) \cdot \nabla \varphi(\mathbf{x})-g(\mathbf{x}) \varphi(\mathbf{x}) \mathrm{d} \mathbf{x}$

for $x=(\lambda, u) \in X$ and $\varphi \in W_{0}^{1, p}(\Omega)$, where $\langle\cdot, \cdot\rangle_{\left(W_{0}^{1, p}\right)^{\prime}, W_{0}^{1, p}}$ denotes the dual pairing between $W_{0}^{1, p}(\Omega)^{\prime}$ and $W_{0}^{1, p}(\Omega)$. Now $f(x)=0$ in $W_{0}^{1, p}(\Omega)^{\prime}$ for $x=(\lambda, u) \in X$ is equivalent with the fact that $u$ is a weak solution to (1) for thickness parameter $\lambda$.

The goal is to determine an optimal thickness parameter $\lambda$ and a corresponding optimal displacement $u$ minimizing the cost functional $J_{f}: X \rightarrow \mathbb{R}$ given by 


$$
J_{f}(x)=\int_{\Omega} \lambda(\mathbf{x})+\frac{\eta}{2}\left|\lambda(\mathbf{x})-\lambda^{\circ}(\mathbf{x})\right|^{2}+\beta \min \left(u(\mathbf{x})-u_{b}(\mathbf{x}), 0\right)^{3} \mathrm{~d} \mathbf{x}
$$

for $x=(\lambda, u) \in X$ subject to (s.t.) the equality constraints $f(x)=0$ in $W_{0}^{1, p}(\Omega)^{\prime}$ and to the inequality constraints $\lambda_{a} \leq \lambda(\mathbf{x}) \leq \lambda_{b}$ f.a.a. $\mathbf{x} \in \Omega$, where $\lambda_{a}, \lambda_{b}$ are positive scalars with $\lambda_{a} \leq \lambda_{b}, \eta \geq 0$ is a regularization parameter and $\lambda^{\circ} \in L^{\infty}(\Omega)$ is a nominal thickness parameter satisfying $\lambda_{a} \leq \lambda^{\circ}(\mathbf{x}) \leq \lambda_{b}$ f.a.a. $\mathbf{x} \in \Omega$. Furthermore, $\beta \geq 0$ is a weighting parameter and $u_{b} \in L^{\infty}(\Omega)$ satisfies $u_{b}(\mathbf{x})>0$ f.a.a. $\mathbf{x} \in \Omega$. The last term of the cost functional $J_{f}$ penalizes the situation if the displacement is larger than the given threshold $u_{b}$. We introduce the set of admissible thickness parameters by

$$
\Lambda_{a d}=\left\{\lambda \in L^{2}(\Omega) \mid \lambda_{a} \leq \lambda(\mathbf{x}) \leq \lambda_{b} \text { f.a.a. } \mathbf{x} \in \Omega\right\}
$$

and define $X_{a d}=\Lambda_{a d} \times\left(W_{0}^{1, p}(\Omega) \cap L^{\infty}(\Omega)\right)$. Then, the infinite-dimensional, nonconvex minimization problem can be formulated abstractly as

$$
\min J_{f}(x) \text { s.t. } x \in \mathcal{F}_{f}=\left\{x \in X_{a d} \mid f(x)=0 \text { in } W_{0}^{1, p}(\Omega)^{\prime}\right\},
$$

where $\mathcal{F}_{f}$ is the set of admissible solutions. Let us refer to $[4,5]$, where a Dirichlet and Neumann optimal control problem governed by the $p$-Laplace equation is considered.

Solving (1) numerically is a difficult task due to the quasilinear elliptic constraint $f(x)=0$ (fine model). In the next section we utilize instead of the accurate, but complex model (1) a linear elliptic PDE as a simpler model that is much easier to solve. Then we combine the simple and the complex model by applying a space mapping approach.

\section{Space mapping}

The space mapping is a mapping between the fine model space parameter or variables and the coarse model space. Then the optimization can be carried out for the coarse model, but information from the fine model is utilized to improve the accuracy of the optimization result with respect to the real application.

As introduced in Section 1 the goal is to replace the fine model (1) by the coarse model (2). Later this fine model will be used in the optimization problem. Existence and uniqueness of a weak solution to (2) were discussed in [3]. Let us now define the Banach space $Y=L^{\infty}(\Omega) \times V$ and introduce the bilinear operator $c: Y \rightarrow V^{\prime}$ (coarse model) by

$$
\langle c(y), \varphi\rangle_{V^{\prime}, V}=\int_{\Omega} 2(1+n) \mu(\mathbf{x}) \nabla v(\mathbf{x}) \mathrm{d} \mathbf{x}-\langle g, \varphi\rangle_{V^{\prime}, V}
$$

for $y=(\mu, v) \in Y$ and $\varphi \in V$. 
Let us now formulate the space mapping. Our fine model is the $p$-Laplace equation (1) with the model output $u$ together with the thickness parameter $\lambda$. The coarse model is given by the linear ellipic PDE (2) with the model output $v$ and the thickness parameter $\mu$. The goal of the space mapping is to adjust the thickness parameter $\mu$ in the coarse model so that the model outputs $u$ and $v$ are similar. Furthermore we want to achieve that the thickness parameters $\mu$ and $\lambda$ are not too distinct.

Concentrating on the region of interest (the subset of $\Omega$, where the force $g$ acts) we consider the space mapping only on $\mathcal{A} \subseteq \Omega$. We define the restriction operator $\mathcal{R}_{\mathcal{A}}: L^{2}(\Omega) \rightarrow L^{2}(\Omega)$ as

$$
\mathcal{R}_{\mathcal{A}} v=v \text { on } \mathcal{A} \text { a.e. } \text { and } \mathcal{R}_{\mathcal{A}} v=0 \text { otherwise. }
$$

Further we introduce the set of admissible thickness parameters by

$$
M_{a d}=\left\{\mu \in L^{2}(\Omega) \mid \mu_{a} \leq \mu(\mathbf{x}) \leq \mu_{b} \text { f.a.a. } \mathbf{x} \in \Omega\right\} \subset L^{\infty}(\Omega) .
$$

Now we introduce the space mapping $\mathcal{P}: \Lambda_{a d} \rightarrow M_{a d}$ as follows: for a given thickness parameter $\lambda \in \Lambda_{a d}$ the corresponding $\mu=\mathcal{P}(\lambda) \in M_{a d}$ is the thickness parameter so that $\mathcal{R}_{\mathcal{A}} v$ is as close as possible to $\mathcal{R}_{\mathcal{A}} u$. We formulate $\mu$ as the solution to a minimization problem. The goal is to determine an optimal thickness $\mu$ for a given $\lambda$ minimizing the cost functional $J_{s p}: Y \rightarrow \mathbb{R}$ given by

$$
J_{s p}(y)=\frac{\gamma}{2} \int_{\mathcal{A}}|v(\mathbf{x})-u(\mathbf{x})|^{2} \mathrm{~d} \mathbf{x}+\frac{\kappa}{2} \int_{\Omega}|\mu(\mathbf{x})-\lambda(\mathbf{x})|^{2} \mathrm{~d} \mathbf{x}
$$

for $y=(\mu, v) \in Y$ subject to $\mu \in M_{a d}$ and the equality constraint $c(y)=0$ in $V^{\prime}$, where $\gamma>0$ is the weighting parameter and $\kappa \geq 0$ is the smoothing parameter.

Let us now formulate the minimization problem more abstractly. The minimization problem can be written as follows

$$
\min J_{s p}(y) \quad \text { s.t. } \quad y \in \mathcal{F}_{s p}=\left\{y \in Y_{a d}=M_{a d} \times V \mid c(y)=0 \text { in } V^{\prime}\right\}, \quad\left(\mathbf{P}_{s p}\right)
$$

where $\mathcal{F}_{s p}$ is the set of admissible solutions.

Assumption 1 For every $\mu \in M_{a d}$ the unique weak solution $v$ to (2) lies in the Hilbert space $H^{s}(\Omega)$ with $s>\max (1, d / 2)$ and

$$
\|v\|_{H^{s}(\Omega)} \leq C\left(1+\|\mu\|_{L^{\infty}(\Omega)}\right)
$$

for a constant $C>0$.

The following theorem ensures existence of optimal solutions to $\left(\mathbf{P}_{s p}\right)$ and states the first-order necessary optimality conditions. For a proof we refer the reader to $[8]$. 
Theorem 2. Let Assumption 1 hold. Then $\left(\mathbf{P}_{s p}\right)$ has at least one optimal solution $y^{*}=\left(\mu^{*}, v^{*}\right) \in Y_{a d}$, which can be characterized by first-order necessary optimality conditions: There exists a unique associated Lagrange multiplier $p^{*} \in V$ together with $y^{*}$ satisfying the adjoint equation

$$
\begin{aligned}
-\operatorname{div}\left(2(1+n) \mu^{*}(\mathbf{x}) \nabla p^{*}(\mathbf{x})\right) & =-\gamma\left(\mathcal{R}_{\mathcal{A}}\left(v^{*}-u\right)\right)(\mathbf{x}) & & \text { f.a.a. } \mathbf{x} \in \Omega, \\
p^{*}(\mathbf{x}) & =0 & & \text { f.a.a. } \mathbf{x} \in \Gamma .
\end{aligned}
$$

Moreover, the variational inequality

$$
\int_{\Omega}\left(\kappa\left(\mu^{*}(\mathbf{x})-\lambda(\mathbf{x})\right)+2(1+n)\left(\nabla v^{*}(\mathbf{x}) \cdot \nabla p^{*}(\mathbf{x})\right)\right)\left(\mu_{\delta}(\mathbf{x})-\mu^{*}(\mathbf{x})\right) \mathrm{d} \mathbf{x} \geq 0
$$

holds for all $\mu_{\delta} \in M_{a d}$.

The optimal control problem given by $\left(\mathbf{P}_{s p}\right)$ can be written in reduced form

$$
\min \hat{J}_{s p}(\mu) \text { s.t. } \quad \mu \in M_{a d} . \quad\left(\hat{\mathbf{P}}_{s p}\right)
$$

The gradient of the reduced cost functional at a given point $\mu \in M_{a d}$ in a direction $\mu_{\delta} \in L^{\infty}(\Omega)$ is given by

$$
\hat{J}_{s p}^{\prime}(\mu) \mu_{\delta}=\int_{\Omega}(\kappa(\mu(\mathbf{x})-\lambda(\mathbf{x}))+2(1+n) \nabla v(\mathbf{x}) \cdot \nabla p(\mathbf{x})) \mu_{\delta}(\mathbf{x}) \mathrm{d} \mathbf{x},
$$

where $v$ satisfies (2) and $p$ solves (4).

We utilize a Newton method with Armijo backtracking line search algorithm $[12$, p. 37$]$ to solve $\left(\hat{\mathbf{P}}_{s p}\right)$. The Newton system

$$
\hat{J}_{s p}^{\prime \prime}\left(\mu^{\ell}\right) d^{\ell}=-\hat{J}_{s p}^{\prime}\left(\mu^{\ell}\right)
$$

is solved by the truncated conjugate gradient (CG) method [12, p. 169]. We find

$$
\left(\hat{J}_{s p}^{\prime \prime}\left(\mu^{\ell}\right) \mu_{\delta}\right)(\mathbf{x})=\kappa \mu_{\delta}(\mathbf{x})+2(1+n)\left(\nabla v_{\delta}(\mathbf{x}) \cdot \nabla p^{\ell}(\mathbf{x})+\nabla v^{\ell}(\mathbf{x}) \cdot \nabla p_{\delta}(\mathbf{x})\right)
$$

f.a.a. $\mathbf{x} \in \Omega$, where $u^{\ell}$ and $p^{\ell}$ satisfy (2) and (4) respectively and $u_{\delta}$ and $p_{\delta}$ satisfy linearized state and adjoint equations; see [8]. Another possibility to solve (5) is to utilize a quasi Newton approximation or the Hessian.

\section{Surrogate optimization}

In this subsection we turn to the surrogate optimization that is used to solve approximately (3). The main idea is to solve the optimization problem using the coarse model $c(y)=0$, but to take the fine model $f(x)=0$ into account by the space mapping technique introduced in Section 3 . 
Let us introduce the Banach space $Z=L^{\infty}(\Omega) \times V$ and the subset $Z_{a d}=$ $\Lambda_{a d} \times V$. We define the cost functional $J_{s o}: Z \rightarrow \mathbb{R}$ as

$$
J_{s o}(z)=\int_{\Omega} \lambda(\mathbf{x})+\frac{\eta}{2}\left|\lambda-\lambda^{\circ}\right|^{2}+\beta \min \left(v(\mathbf{x})-u_{b}(\mathbf{x}), 0\right)^{3} \mathrm{~d} \mathbf{x}
$$

for $z=(\lambda, v) \in Z$, where $\eta, \lambda^{\circ}, \beta, u_{b}$ are as in Section 2. We consider the optimization problem

$$
\min J_{\text {so }}(z) \quad \text { s.t. } \quad z \in \mathcal{F}_{\text {so }}
$$

where the feasible set is

$$
\mathcal{F}_{\text {so }}=\left\{z=(\lambda, v) \in Z_{a d} \mid c(\mu, v)=0 \text { and } \mu=\mathcal{P}(\lambda)\right\} .
$$

We suppose that $\left(\mathbf{P}_{s o}\right)$ has a local optimal solution $z^{*}=\left(\lambda^{*}, v^{*}\right) \in Z_{a d}$. In particular, we have $v^{*}=\mathcal{S}_{c}\left(\mathcal{P}\left(\lambda^{*}\right)\right)$, where $\mathcal{S}_{c}$ denotes the solution operator for the coarse model. The corresponding reduced problem is given by

$$
\min \hat{J}_{s o}(\lambda) \quad \text { s.t. } \quad \lambda \in \Lambda_{a d}
$$

with

$$
\hat{J}_{s o}(\lambda)=\int_{\Omega} \lambda(\mathbf{x})+\frac{\eta}{2}\left|\lambda-\lambda^{\circ}\right|^{2}+\beta \min \left(v(\mathbf{x})-u_{b}(\mathbf{x}), 0\right)^{3} \mathrm{~d} \mathbf{x}, \quad \lambda \in \Lambda_{a d} .
$$

with $v=\mathcal{S}_{c}(\mathcal{P}(\lambda))$. Next we state the first-order necessary optimality conditions for $\left(\mathbf{P}_{\text {so }}\right)$.

Theorem 3. Let Assumptions 1 hold. Suppose that $z^{*}=\left(\lambda^{*}, v^{*}\right)$ is a local solution to $\left(\mathbf{P}_{\text {so }}\right)$. Then there exist unique associated Lagrange multipliers $p^{*} \in V$ and $\xi^{*} \in L^{2}(\Omega)$ together with $z^{*}$ satisfying the adjoint equation

$$
\begin{array}{rlrl}
-\operatorname{div}\left(2(1+n) \mu^{*}(\mathbf{x}) \nabla p^{*}(\mathbf{x})\right) & =-3 \beta \min \left(v^{*}(\mathbf{x})-u_{b}(\mathbf{x}), 0\right)^{2} & & \text { f.a.a. } \mathbf{x} \in \Omega, \\
p^{*}(\mathbf{x}) & =0 & \text { f.a.a. } \mathbf{x} \in \Gamma .
\end{array}
$$

Moreover, the variational inequality

$$
\begin{array}{r}
\int_{\Omega}\left(1+\eta\left(\lambda^{*}(\mathbf{x})-\lambda^{\circ}(\mathbf{x})\right)+2(1+n) \mathcal{P}^{\prime}\left(\lambda^{*}\right)^{\star}\left(\nabla v^{*}(\mathbf{x}) \cdot \nabla p^{*}(\mathbf{x})\right)\right) \\
\left(\lambda_{\delta}(\mathbf{x})-\lambda^{*}(\mathbf{x})\right) \mathrm{d} \mathbf{x} \geq 0
\end{array}
$$

holds for all $\lambda_{\delta} \in \Lambda_{a d}$, where $\mathcal{P}^{\prime}\left(\lambda^{*}\right)^{\star}$ denotes the adjoint operator to $\mathcal{P}^{\prime}\left(\lambda^{*}\right)$.

It follows that the gradient $\hat{J}_{\text {so }}^{\prime}$ of the reduced cost functional is given by

$$
\hat{J}_{\text {so }}^{\prime}(\lambda)=1+\eta\left(\lambda-\lambda^{\circ}\right)+\mathcal{P}^{\prime}(\lambda)^{\star} 2(1+n) \nabla v(\cdot) \cdot \nabla p(\cdot) \quad \text { in } \Omega,
$$

where function $v$ satisfies 


$$
\begin{aligned}
-\operatorname{div}(2(1+n) \mu(\mathbf{x}) \nabla v(\mathbf{x})) & =g(\mathbf{x}) \\
v(\mathbf{x}) & =0 \quad \text { f.a.a. } \mathbf{x} \in \Omega
\end{aligned}
$$

with $\mu=\mathcal{P}(\lambda)$ and $p$ is the solution to

$$
\begin{array}{rlrl}
-\operatorname{div}(2(1+n) \mu(\mathbf{x}) \nabla p(\mathbf{x})) & =-3 \beta \min \left(v^{*}(\mathbf{x})-u_{b}(\mathbf{x}), 0\right)^{2} & \text { f.a.a. } \mathbf{x} & \in \Omega, \\
p(\mathbf{x}) & =0 & \text { f.a.a. } \mathbf{x} \in \Gamma .
\end{array}
$$

To avoid the computation of the operator $\mathcal{P}^{\prime}(\lambda)$ we apply Broyden's updating formula providing a matrix $B$ which can be used to replace $\mathcal{P}^{\prime}(\lambda)$, but also $\mathcal{P}^{\prime}(\lambda)^{\star}$. We use a modified Broyden's update formula introduced in [6]. This update formula is given by

$$
B_{\ell+1}=B_{\ell}+\frac{\widetilde{\mathcal{P}_{\delta}}-B_{\ell} \lambda_{\delta}}{\left\|\lambda_{\delta}\right\|_{L^{2}(\Omega)}^{2}}\left\langle\lambda_{\delta}, \cdot\right\rangle_{L^{2}(\Omega)}
$$

with

$$
\widetilde{\mathcal{P}_{\delta}}=\mathcal{P}_{\delta}+\sigma \frac{\hat{J}_{\delta}-\left\langle\hat{J}_{\text {sur }}^{\prime}\left(\lambda^{k}\right), \mathcal{P}_{\delta}\right\rangle_{L^{2}(\Omega)}}{\left\|\lambda_{\delta}\right\|_{L^{2}(\Omega)}^{2}} \hat{J}_{\text {sur }}^{\prime}\left(\lambda^{\ell}\right),
$$

where $\hat{J}_{\delta}=\hat{J}_{\text {so }}^{\prime}\left(\lambda^{\ell+1}\right)-\hat{J}_{\text {so }}^{\prime}\left(\lambda^{\ell}\right), \lambda_{\delta}=\lambda^{\ell+1}-\lambda^{k}$ and $\mathcal{P}_{\delta}=\mathcal{P}\left(\lambda^{\ell+1}\right)-\mathcal{P}\left(\lambda^{\ell}\right)$. Note that for $\sigma=0$ we get the classical Broyden's update formula.

For the numerical solution we apply the gradient projection method using Broyden's updating to obtain an approximation of the sensitivity $\mathcal{P}^{\prime}(\lambda)$.

\section{Numerical results}

In this section we present numerical results for the space mapping and the surrogate optimization. For our numerical example we consider a domain representing a simplified door, denoted by $\Omega$. The red line in Figure 2 (left plot) indicates the section of the boundary, where homogeneous Neuman boundary conditions of the form $\langle\nabla u(x), \vec{n}\rangle_{2}=0$ are applied, where $\vec{n}$ denotes an outer normal on the boundary and $\langle\cdot, \cdot\rangle_{2}$ the Euclidean inner product. We use the finite element discretization and solvers for (1) and (2) provided by the Matlab Partial Differential Equation Toolbox. The right-hand side $g(\mathbf{x})$ (force term) is given as follows:

$$
g(\mathbf{x})=\left\{\begin{array}{c}
47.71, \mathbf{x} \in \mathcal{B}_{r}\left(\mathbf{x}_{\text {mid }}\right)=\left\{\mathbf{x} \in \Omega|| \mathbf{x}_{\text {mid }}-\left.\mathbf{x}\right|_{2}<r\right\} \\
\text { 0, otherwise, }
\end{array}\right.
$$

where $\mathbf{x}_{\text {mid }}=(0.5,0.45)^{T}$ and $r=0.1$. This force term is indicated as the inner circle in Figure 2 (left plot). Let us next state the parameters for our numerical example. The Hollomon coefficient is set to $n=0.22$. For the space mapping we choose the weight parameter as $\gamma=\left(\int_{\Omega}|u(\mathbf{x})|^{2} \mathrm{~d} \mathbf{x}\right)^{-1}$ and $\kappa=$ $10^{-3} \gamma$. Further we choose the region $\mathcal{A}$ to be a circle with radius 0.2 and 
midpoint $(0.5,0.45)$, illustrated in Figure 2 (left plot) by a green circle. Next we have a look at the parameters for the surrogate optimization. We choose $\eta, \beta$ and $\lambda^{\circ}$ to be $1.25,25^{5}$ and 1.7 , respectively. The threshold $u_{b}$ is set to 0.3 and the bounds for the thickness parameter are set to $\mu_{a}=\lambda_{a}=0.05$ and $\mu_{b}=\lambda_{b}=10$. As a stopping criteria we choose the norm of the reduced gradient to be smaller than 0.1 times the maximum diameter of the finite elements. We will report on numerical results for two different settings for the parameter $\sigma$.
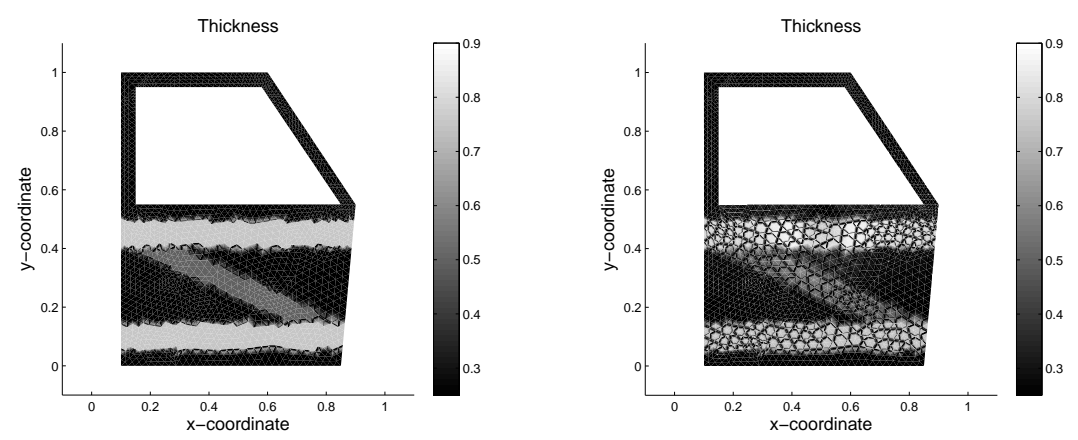

Fig. 1. Initial thickness parameter (left plot) and the optimal thickness parameter $\mu^{*}$ (right plot) for the space mapping using the Newton-CG method.
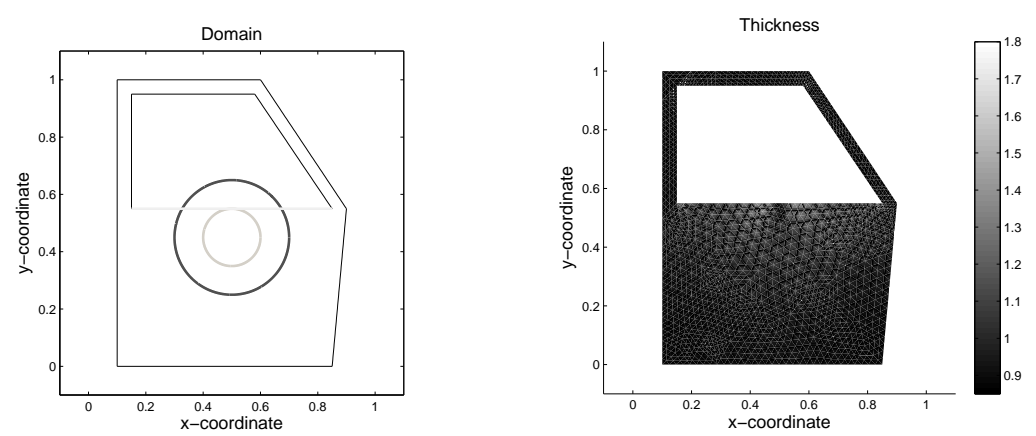

Fig. 2. Domain $\Omega$ (left plot) and the optimal thickness parameter $\lambda^{*}$ (right plot) for the surrogate optimization.

Let us first present a numerical result for the space mapping. As an initial thickness for the space mapping we choose a structured initial thickness parameter, shown in Figure 1 (left plot). In the right plot of Figure 1 we present the corresponding thickness parameter $\mu^{*}$ computed by the space mapping. We observe that the thickness parameter is enlarged in the region $\mathcal{A}$. In Table 

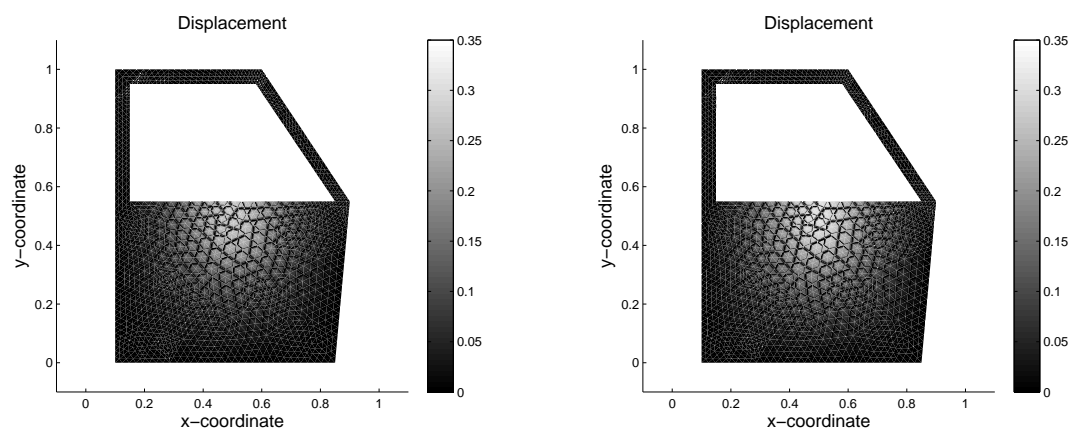

Fig. 3. Displacement $v$ of the linear model (left plot) and $u$ of the $p$-Laplace equation (right plot) for the optimal thickness parameter $\lambda^{*}$.

Table 1. Summary of the results for the space mapping and the performance for two different methods.

\begin{tabular}{|c|c|c|c|c|}
\hline & $v$ & $u$ & BFGS & Newton-CG \\
\hline $\max _{\Omega}$ & 0.68961 & 0.59601 & 0.59541 & 0.59462 \\
\hline Iterations & & & 9 & 4 \\
\hline Time (sec) & & & 8.52 & 4.81 \\
\hline
\end{tabular}

Table 2. Summary of the results for the surrogate optimization and the performance of the gradient projection method for two different Broyden's updates ( $\sigma=0$ and $\sigma=0.2$ ).

\begin{tabular}{ccccccccc}
\hline$\sigma$ & $\max _{\Omega} u$ & $\max _{\Omega} v$ & Volume & $\min _{\Omega} \lambda$ & $\max _{\Omega} \lambda$ & $\|u-v\|_{L^{2}(\Omega)}$ & Iter & Time (sec) \\
\hline 0.0 & 0.31307 & 0.27650 & 0.48857 & 0.89759 & 1.77613 & 0.01198 & 10 & 82.72 \\
0.2 & 0.31313 & 0.27606 & 0.48899 & 0.89555 & 1.67856 & 0.01204 & 7 & 57.65 \\
\hline
\end{tabular}

1 the numerical results and performace for the space mapping utilizing the Newton-CG and the BFGS algorithms are summarized. It turns out that for the thichness parameter shown in Figure 1 (left plot) the maximal displacements for $v$ (solution to the linear model) and $u$ (solution to the $p$-Laplacian) are quite different. Using the space mapping the optimal thickness parameter leads to a maximal displacement in the linear model that is very close to maximal one of $u$. Furthermore, we observe from Table 1 that the NewtonCG method performs significantely better then the BFGS method while giving nearly the same results measured in the maximum displacement.

Next we present the numerical results for the surrogate optimization. In Figure 2 (right plot) the optimal thickness parameter $\lambda^{*}$ for the surrogate optimization is shown. The corresponding displacements for the coarse and fine model are shown in Figure 3 (left and right plot), respectively. In Table 2 we summarize the numerical results for the two different values for $\sigma$. Note that 
the modified Broyden's update give a better performance than the classical Broyden's update with respect to the number of iterations and CPU time while giving nearly the same results.

\section{References}

1. M.H. Bakr, J.W. Bandler, K. Masden, and J. Søndergaard. An introduction to the space mapping technique. Optimization and Engineering, 2(4):369-384, 2001.

2. J.W. Bandler, R.M. Biernacki, Shao Hua Chen, P.A. Grobelny, and R.H. Hemmers. Space mapping technique for electromagnetic optimization. IEEE Transactions on Microwave Theory and Techniques, 42(12):2536-2544, 1994.

3. E. Casas. Optimal control in coefficients of elliptic equations with state constraints. Applied Mathematics and Optimization, 26(1):21-37, 1992.

4. E. Casas and L.A. Fernández. Distributed control of systems governed by a general class of quasilinear elliptic equations. Journal of Differential Equations, 104(1):20-47, 1993.

5. E. Casas and L.A. Fernández. Dealing with integral state constraints in boundary control problems or quasilinear elliptic equations. SIAM Journal on Control and Optimization, 33(2):568-589, 1995.

6. M. Hintermüller and L.N. Vicente. Space mapping for optimal control of partial differential equations. SIAM Journal on Optimization, 15(4):1002-1025, 2005.

7. O. Lass, C. Posch, G. Scharrer, and S. Volkwein. Space mapping techniques for the optimization of a thickness parameter in the p-laplace equation. Submitted, 2009 .

8. O. Lass. Efficient numerical space mapping techniques for the $p$-Laplace equation. Diploma thesis, Institut für Mathematik und Wissenschaftliches Rechnen, Karl-Universität Graz, in preparation, 2009.

9. S.J. Leary, A. Bhaskar, and A.J. Keane. A constraint mapping approach to the structural optimization of an expensive model using surrogates. Optimization and Engineering, 2(4):385-398, 2001.

10. J. Marburger. Space Mapping and Optimal Shape Design. Diploma Thesis, TU Kaiserslautern, 2007.

11. H. Maurer and J. Zowe. First and second order necessary and sufficient optimality conditions for infinite-dimensional programming problems. Mathematical Programming, 16(1):98-110, 1979.

12. J. Nocedal and S.J. Wright. Numerical optimization. Springer Series in Operations Research, Springer-Verlag, New York, 2006.

13. G. Scharrer, S. Volkwein, and T. Heubrandtner. Mathematical optimization of the plate volume under a p-laplace partial differential equation constraint by using standard software. Submitted, 2009.

14. L.N. Vicente. Space mapping: models, sensitivities, and trust-region methods. Optimization and Engineering, 4(3):159-175, 2003. 